\title{
HOMOERÓTICAS: TEORÍA Y APLICACIONES
}

\author{
David William Foster
}

\begin{abstract}
RESUMEN
El examen de la producción cultural relacionada con el problema del homoerotismo no puede ser emprendido sin una adecuada base teórica. Esta base necesariamente tiene que ver con la definición de las relaciones entre lo homoerótico y la ideología hegemónica de heterosexualidad compulsiva que sustenta al patriarcado. Además, implica la definición de lo que se entiende por homoerotismo y de cómo una posición sociocultural se opone al patriarcado y proporciona una serie de principios conceptuales y estratégicos que pueden generar una producción cultural contestataria de resistencia. Finalmente, se ofrece una discusión acerca de qué podrían manifestar algunas de las dimensiones de esta producción cultural y de cuáles podrían ser las características particulares de estas en América Latina.
\end{abstract}

\section{ABSTRACT}

The examination of Latin American cultural production relating to issues of homoeroticism cannot be undertaken without an adequate theoretical base. This base necessarily concerns itself with the definition of the relationship between the homoerotic and the hegemonic ideology of compulsory heterosexuality that sustains the patriarchy. Concomitantly, it envolves the definition of what is understood by homoeroticism, and how such a sociocultural positioning responds to the patriarchy and furnishes a series of conceptual and strategic principles that are able to generate a resistant and contestatorial cultural production. Finally, one offers a discussion of what some of the dimensions of that cultural production might manifest and what the particular characteristics of its occurrence in Latin American might constitute.

"Orden en el desorden

La succión se aprende en el pezón del seno materno

o en la mamila, después se practica con el chupón o en el dedo pulgar.

Lo que siga, ya es cuestión del azar." Elías Nandino, Erotismo al rojo blanco

“(...) de ángeles amándose jamás se construyó una tumba y un tálamo sin alas de alas “¿para quién? ¿de machos necios que acusáis al maricón sin razón, sin ver que sois la ocasión de lo mismo que culpáis?"

Manuel Ramos Otero, El libro de la muerte 
Son dos las características descollantes de lo homoerótico en lo que se refiere a llevarlo hacia una teorización cultural:

1) Lo homoerótico se fundamenta en una epistemología abierta que repudia las definiciones fijas sobre las que se tensa el patriarcado y sus definiciones de la sexualidad. Fijar la lengua -y de ahí fijar el mundo -, siempre ha sido el sueño rector del patriarcado; y uno de los impulsos cruciales de lo homoerótico es la subversión de este proyecto en aras de otras maneras de construir una epistemología de la experiencia y la subcategoría que de ella constituye la sexualidad.

2) En vista de que el patriarcado propone un sistema cerrado de análisis social e histórico, tanto en lo que respecta a lo que excluye como en lo referente a sus aspiraciones hacia un modelo que lo engloba y lo explica todo, lo homoerótico se sustrae de la urgencia de formular un contramodelo igualmente excluyente y englobante. Lo homoerótico no constituye una narrativa maestra, ni se propone elaborar tal cosa pues busca dejar abiertas y en suspenso consideraciones sobre identidades fijas, motivaciones enteramente consecuentes, antecedentes y procedentes estrictamente unidireccionales y transitivos y formulaciones exclusivamente entrelazadas. Se trata de una sana duda en cuanto a la posibilidad de entender el vasto maremagnum del deseo humano y de compulsar adecuadamente una supuesta coherencia en todos los aspectos de las interrelaciones eróticas de subjetividades sumamente volubles.

Si el patriarcado y su ideología dominante, el heterosexismo compulsivo, se fundamentan en esquemas promulgados como universales y sempiternos, le incumbe a lo homoerótico manejar una ética del conocimiento que admita la más amplia variedad posible de interpretaciones y de elaborar modelos de conocimiento que puedan romper con el autoritarismo, tanto el epistemológico como el sociopolítico (anverso y reverso de la misma moneda en cuanto a cómo se sustentan mutuamente). Conformarse con las limitaciones en cuanto a lo que se puede conocer y prescindir de las tecnologías de control que permite el sistema patriarcal, son principios claves de lo homoerótico.

Lo homoerótico, por ende, propone abrir un debate, no cerrarlo. Bien que haya la posibilidad y la conveniencia estratégica, de hacer propuestas interpretativas que puedan guiar movimientos y programas, lo homoerótico propone sustraerse del proyecto de encontrar la Verdad de los constructos sociales. Mucho más eficaz es el cuestionamiento de los constructos, no necesariamente para rebatirlos sino para investigar sobre las fuerzas sociales e históricas que los han creado y sostenido y a efectos de qué postulaciones ideológicas. Si la idea de la conclusión contundente arrojada por un riguroso programa de investigación fundamentada en una metodología coherente es un ideologema del patriarcado y su afán por el control tecnológico de la sociedad, lo homoerótico, sin obligarse a rechazar airadamente tales propuestas, se concede el derecho de subrayar su estatus como un idelogema dominante y de proponer perspectivas alternativas para el análisis de lo social.

El patriarcado funciona sobre la base de una estricta homología entre másculo (macho)masculino-hombre y femíneo (hembra)-femenino-mujer; concomitantemente maneja una serie de propuestas que tienden a demostrar que la segunda conjugación se subordina a la primera y, 
de algún modo, deriva de ella -lo femenino entendido como una inversión distorsionada de lo masculino o como una versión deficiente o en carencia: el sistema ginecológico como los genitales masculinos invertidos; la vagina como signo de la castración del pene y los testículos o el clítoris como una atrofia (¿parodia?) del miembro viril.

En las dos conjugaciones, el primer término alude a la condición biológica (vista como predeterminada e inapelable- por ello, imperativamente inalterable). El segundo se refiere a la identidad de género que se forja sobre la base del sexo biológico. Visto como un conjunto de caracteres inamovibles, se practica una distribución incondicional de cada género frente a las categorías sexuales. En el discurso popular, se tiende a tratar sexo y género como sinónimos, tanto las palabras como las categorías que en esta formulación se pretende captar. Dicha sinonimia refuerza la continuidad sin fisuras entre las esferas del constructo social del patriarcado y los principios del heterosexismo compulsivo. El tercer término apunta al papel social que los cuerpos están conminados a cumplir, papeles que son proyectos de toda una vida. La contrucción de cada uno se vale del amplio panorama de elementos de la tecnología de la identidad genérica y, el individuo, a lo largo de toda la vida, se afana en pulir, mantener y corregir la realización de uno y otro de los únicos dos papeles válidos. Ser "nada menos que todo un hombre" o "nada menos que toda una mujer" requiere un enorme esfuerzo y múltiples convalidaciones frente a uno mismo y frente a los otros. De más está decir que no muchos cumplen con un papel estelar y pocos triunfan sin ambages. Sólo en la tercera edad se encuentran concesiones y treguas, cuando no directamente un grado de hastío, como la mujer que comienza a negarse a la máscara del maquillaje o el hombre que acepta ir de compras en el barrio con una bolsa de mercado de plástico. Revisar este sistema de homologías no conducirá imperiosamente a una configuración definitiva de la sociedad, pero sí ofrecerá una brecha desde la cual se puede contemplar la dinámica constructiva del patriarcado. En una primera instancia, una revisión permitirá entender la inestabilidad esencial de este sistema donde, una y otra vez, en la realidad de la sociedad vivida casi sin excepciones, el individuo deja de cumplir, de alguna u otra manera, con este entramado y con las delicadas negociaciones en el fuero cultural que le permiten sostener una afinada homología entre los componentes. Se enfatiza que, por lo menos a los efectos de la discusión en este punto, se está hablando de frustradas tentativas o de irreflexivos incumplimientos con el sistema homológico del patriarcado. La histeria que aureola el discurso hegemónico de la sexualidad se deriva de las dificultades, en el diario vivir, de cumplir con las exigencias impuestas, y se postula que gran parte de la violencia social proviene del enorme sistema de vigilancia, denuncia, corrección y castigo que hace falta para asegurar, aunque sólo con un alto grado de ineficacia, que los individuos adhieran y atiendan a su cumplimiento.

La propuesta que se ha hecho respecto de la división tripartita de las homologías rectoras del patriarcado, se propone captar las tres fases en la construcción de la identidad sexual. Lo que importa subrayar es cómo esta relación no es exclusivamente unidireccional, en el sentido de que el sexo determina el género y el género determina el papel social. En varias etapas de la formación del sujeto, tanto durante el período en que está férreamente sometido y circunscrito por las tecnologías que administran las instituciones del patriarcado (familia, escuela, cancha, club/asociación social, guarnición), como durante el transcurso de toda su vida, hay un movimiento de iteración, reduplicación, confirmación, validación entre cada una de las esferas, para que lo biológico, lo subjetivo y lo actuado (es decir, el papel social que se juega) se identifiquen y con- 
firmen mutuamente. Quien se llame Concepción tiene que tener una matriz y tiene que dar a luz hijos; tener una matriz y dar a luz hijos y llamarse Manolo, en cambio, perturba seriamente el sistema de homologías. Por ello, la bisexualidad suele ser más detonante que la transexualidad, en la medida en que ésta, salvedad hecha del escándalo de la reconfiguración puntual del cuerpo, mantiene esencialmente el sistema homológico del patriarcado, mientras que aquélla desafía radicalmente el imperativo de un reparto binario fijo.

En una segunda instancia, la revisión de las conjugaciones homológicas abre paso a maneras para el individuo de sustraerse de las rígidas homologías del patriarcado, sustraerse no tanto para cumplir con un programa de reconfiguración de su identidad sexual (travestismo, transexualidad, bisexualidad y homoerotismo, amén de disidencias parciales y menos determinantes), sino para entender en qué formas puede dejar de colaborar con ellas, en qué modos no importa si las cumple rigurosamente o no. El precio para tales excepciones suele ser alto y no pocas veces sangriento, pero para muchos el beneficio de asumir cierta conducción más personal en la navegación de la sexualidad vale el costo. Importa recalcar que aquí no se está hablando de efectuar la propuesta de una nueva configuración de homologías para la sociedad, de descubrir una nueva "verdad" en cuanto a sus interrelaciones, sino de colocar sobre el tapete la posibilidad de contemplar múltiples reformulaciones y las consecuencias, tanto negativas como positivas, que puedan obtenerse.

Lo homoerótico se erige contra el heterosexismo compulsivo. No contra el heterosexismo en sí: por grande que sea la norma, no deja de ser una opción entre otras (p.ej., la ménage à trois). Se puede o no optar por el heterosexismo, y se puede optar por él con exclusión de otras opciones o en concierto con ellas. El heterosexismo será una opción normalizadora por razones de recreación biológica y/o como consecuencia del peso de una determinada herencia socio-culrural plasmada en múltiples ideologías políticas y religiosas. Pero la compulsión hacia el heterosexismo opera a fin de excluir cualquier otra opción disyuntiva o coyuntiva y se fundamenta más que nada en la premisa de que cualquier apartamiento de él amenaza la reproducción de la raza humana, de que la raza humana sólo puede perpetuarse mediante una rigurosa imposición y el mantenimiento del heterosexismo compulsivo. La compulsión al heterosexismo trae a colación otro sistema de relaciones homológicas. Impone la necesidad del matrimono monogámico y reproductivo. El individuo que no se casa, si no es por razones sancionadas por el Estado (sea la categoría privilegiada de los desposados con un Ser Supremo o sea la categoría de la escoria marginada: prostitutas, presos o deficientes), ocupa una Tierra de Nadie, cuando no es blanco de francos repudios sociales. Y la pareja que no se reproduce, si no es por razones confirmadas por la ciencia (infertilidad, inaptitudes fisiológicas), queda igualmente estigmatizada. A veces la relación homoerótica se denuncia menos por lo que se pueda suponer que es (y siempre se supone que aparece acompañada de una total exclusión de una relación heteroerótica paralela), que por lo que no es, en lo que al aspecto reproductivo se refiere: se trata de desperdiciar la simiente.

La estricta correlación entre reproducción y monogamia, que la asegura y legitima dentro de una genealogía social, se complementa con la estricta correlación entre romance, intimidad, amor y placer. Sólo la pareja heterosexual que logra la armonía íntegra entre estas (y posiblemente otras) esferas de emociones o sentimimientos cumple con el paradigma del heterosexismo. Concomitantemente, cualquier sustracción a esta homología atenta contra la ley del 
heterosexismo y abre paso a la disidencia/disolución sexual. Dentro de una concepción de lo homoerótico que reconozca la posibilidad del placer erótico sin el amor romántico -o de una relación sentimental que excluya la reproducción de la especie- se ve asomar el enemigo del heterosexismo compulsivo. Por mucho que hoy se admita, en mayor o menor medida, la congruencia de prácticas no reproductivas dentro de la pareja, es siempre en busca de consolidar otras dimensiones de la relación que tenderán a propiciar la reproducción en su debido momento y las responsabilidades familiares que ella acarrea.

Hay sectores sociales dispuestos a tolerar las relaciones homoeróticas "a falta de algo mejor" (i.e., una pareja del sexo opuesto), por la imposibilidad de mantener una relación heterosexual, la cual es considerada como el punto de referencia de la sociedad. Por ello, se tiende a contemplar con benevolencia aquellas relaciones homoeróticas que se asemejan a un paradigma de la relación heterosexual y a negar la legitimidad, o por lo menos, el estatus ontológico de cualquier relación que se resista a tal equiparencia.

La lectura de los textos de la producción cultural siempre acarrea serios problemas en cuanto a la interpretación de la relación entre los personajes y el mundo de la "realidad social", configurado como exterior al texto, pero al que éste apela con uno que otro grado de coherencia e identificación. Nuestra tradición lectora da por supuesto que, en el caso de los discursos en primera persona, hay una estrecha relación autobiográfica entre autor y narrador. Esto, no tanto porque el autor tenga la necesidad de disfrazarse por miedo a las represalias homofóbicas, sino porque si se alega que el discurso autorreferencial, aún más que cualquier otro tipo de discurso, supone la configuración de una realidad y no un mero reportaje, se entiende cómo el narrador pueda estar refiriendo un deseo y renarrando experiencias vividas.

Esta problematización de la relación entre autor y narrador se complica en otra dimensión si se cuestiona la unilateralidad sexual (un hombre sólo habla en cuanto hombre, una mujer sólo habla en cuanto mujer, un indígena sólo habla en cuanto indígena, un/a gay sólo habla en cuanto gay) o hasta si se cuestiona el presupuesto de que los narradores tienen sexo: el marcador gramatical del narrador que alude a sí mismo no es necesariamente el conjunto de culturemas que evoca al sistema social de la sexualidad y el género cuando aparece usado por una persona en el diario vivir fuera de las convenciones de la literatura.

La tentativa de perfilar rasgos determinantes para una reproducción cultural lesbiana o gay tiene que fracasar en cuanto la naturaleza proteica de esta producción en una sociedad (pos)moderna, se resiste a cualquier tipo de limitación a priori. Sin embargo, en un momento específico y en una cultura específica, es posible detectar aglomeraciones de ciertas modalidaes de producción que se relacionan con (aún cuando no son la consecuencia de) líneas de fuerza en el debate público al que responde en parte la producción cultural.

De ahí que hayan surgido formas expresivas privilegiadas: planteos autobiográficos (contar la histora personal de los esfuerzos por separarse de la ideología del patriarcado y de forjar una subjetividad propia), picarescos (las experiencias contestatarias del individuo en los márgenes e intersticios de la sociedad decente), confesionales (aunque la confesión es una de las grandes tecnologías del patriarcado, dar la vuelta para confesar lo que no se debe confesar, lo que se alega no existe, para exculparse de lo que no tiene exculpación, es crear un contradiscurso que si no es privativo de lo homoerótico, caracteriza a todos los movimientos de reivindicación social). Otra modalidad como el diario representa un decir, en la 
penumbra de la escritura en que el formato facilita, en un texto privado del vehículo público de la novela, lo que no debe decirse.

O en otro registro, ha habido esfuerzos para minar las posibilidades de la pornografía, pero menos para complementar la que ya existe con el inventario de la pornografía lesbiana o gay, que para practicar una auscultación del concepto mismo de pornografía. Se ha dicho que la pornografía es la teoría y la violación es la práctica, entendiendo que los dos son instrumentos del patriarcado en su afán por mantener fijos los papeles sexuales y castigar cualquier desviación de ellos. Esa formulación se fundamenta en la observación de que la pornografía es esencialmente un género masculino y los partícipes son esencialmente mujeres que sufren vejaciones a manos de los hombres. En ese sentido, la pornografía modela lo que le pasa a la mujer si se aparta del camino y de la protección del padre, al mismo tiempo que le concede a los hombres el derecho de valerse hasta el máximo del cuerpo de la mujer que se encuentra desvalido del amparo paterno. En una pornografía homoerótica en la que se conserven incólumes las estructuras del patriarcado, nada cambia en términos de esta formulación.

Pero muy otras son las cosas en una producción pornográfica que se propone enfrentarse con el patriarcado, o en aras de la liberación femenina o en aras del homoerotismo transgresor (a veces en estos casos se habla de la erótica, al insistir en que ésta rompe con los esquemas repetitivos e irreflexivos de la pornografía, como si las divisiones pudieran hacerse tan nítidamente). La exposición del cuerpo, visual o verbalmente, comprometido con prácticas negadas y repudiadas por el patriarcado, crea un campo de producción cultural privilegiada en lo que a revisar esquemas discursivos se refiere, un campo donde se puede suspender radicalmente la distinción clave, por ejemplo, entre quién hace y quién se deja hacer.

Se subraya cómo una lectura de la producción cultural es siempre política o ideológica. De ahí el énfasis psicológico, personal y moral de esta producción y de los análisis de la misma, constituye una postura ideológica. El grado en que un texto explicite esto o el grado en que un análisis crítico lo pueda enmarcar, fomenta una consideración de cuáles son los efectos que un determinado discurso cultural produce en determinados lectores, como individuos o como comunidades, de cuáles serán los horizontes de conocimiento y las aspiraciones al conocimiento de estos grupos, y de cuáles serán sus prácticas para una lectura de una narrativa de una específica línea temática o de una específica modalidad literaria. Al mismo tiempo, hay que reconocer que sigue habiendo una separación bastante pronunciada entre la producción cultural "académica" que llega a un público relativamente restringido y una producción cultural, ampliamente definida, que constituye el consumo básicamente convencionalizado de la masa de lectores. Mientras que se suelen encontrar en la primera propuestas para la innovación y la resistencia, ésta última pone en circulación un discurso humorístico/satírico abundantemente cargado de referencias sexuales, entre ellas homosexuales. Sin embargo, la concepción de la subjetividad en la que descansan estas referencias no pasa de ser, en el fondo, una reconfirmación de las leyes del juego del patriarcado, sin ningún tipo de reflexión suficiente para cuestionarlo. Puede haber hasta cierto punto una acumulación de raspaduras en la fachada del patriarcado, pero sería arriesgado confundir tales arranques muy parciales y esencialmente circunspectos con el grado de revisión radical de las estructuras del patriarcado que contemplan las propuestas contestatarias. Y una lectura homoerótica, vista como un imperativo ético o como un imperativo político, con todas las graduaciones que permiten estas dos opciones fundamentales, es necesariamente una prácti- 
ca radical, en la medida en que el heterosexismo compulsivo sigue siendo la norma cultural irreflexiva e incuestionada de nuestra sociedad.

La cultura homoerótica necesariamente pasa por los espacios urbanos. Aunque es lógico que haya homoerotismo en todo espacio vivencial, la identidad de algo como una "cultura gay" y el desarrollo de una teorización y una ideología de una cultura gay, están íntimamente vinculados al entorno metropolitano. Esto se debe, en parte, a que las grandes instituciones del patriarcado son también metropolitanas y, en parte, al hecho de que el abanico de intereses culturales en las grandes concentraciones urbanas crea situaciones propicias para el análisis de las estructuras hegemónicas y para la exploración de vivencias alternativas. De hecho, las grandes ciudades siempre han servido de refugio para individuos que no puedan conformarse al peso más notable del patriarcado en el campo o en la pequeña urbe (la contrapartida de esto es que, si es cierto que las instituciones del patriarcado tiene su centro de poder en la ciudad, puede resultar más sencillo en el campo desaparecer del escrutinio de la hegemonía).

La ciudad proporciona varias oportunidades para el refugio o el resguardo, comenzando por el hecho de que las grandes concentraciones demográficas son más difíciles de vigilar y así, el ciudadano prevenido puede ejercer más libertad que en otros lugares. Segundo, la vida urbana permite muy fácilmente la concentración de personas en sectores (barrios, guetos, distritos, suburbios) donde hay cierta afinidad de intereses e identidades. Serán hasta cierto punto sectores de una "permisividad alambrada", frente a los que la conciencia pública general (dominante) funciona con estereotipos y cierta actitud de indulgencia paternalista, pero el simple hecho es que gran parte de la producción cultural sobre las minorías culturales proviene de tales sectores.

Así mismo, la internacionalización de los centros urbanos de América Latina ha significado la asimilación de conceptos y prioridades de otras culturas, los cuales están a veces en conflicto con la cultura nacional(ista). Esto se nota en que un sistema latinoamericano (de origen hispánico y mediterráneo en general) donde la categoría de "homosexual", tradicionalmente limitada a la pareja pasiva de un acto homoerótico, está cediendo paso, o por lo menos confrontándose con las propuestas modernas respecto de la identidad inherente del individuo (uno es homosexual por lo que siente que es y no por los actos que realiza o su papel en aquellos actos) o posmodernas (el cuestionamiento de las bases epistemológicas del conocimiento -en este caso, el conocimiento en cuanto a qué son los actos y qué son los papeles dentro de los actos). Es probable que en los centros más "sofisticados" de América Latina, las propuestas de la identidad y las desconstrucciones de las categorías vayan a terminar prevaleciendo, conforme con los modelos extranjeros e internacionales que están impactando, en parte gracias a los medios masivos de comunicación y gracias a los esfuerzos de la política de la democracia institucional de buscar nuevos modelos. Lo último es en particular del orden del día en aquellas sociedades que tienen todavía una memoria de la tiranía militar y de los vínculos entre las fuerzas armadas de la represión y la ley del patriarcado.

Cualquier intento de entablar una discusión acerca de una herencia lesbiana y gay para América Latina, en forma inevitable e inmediata, remite al problema de las culturas dominantes, tales como las de los principales centros mundiales (generalmente europeos) y las de grupos establecidos reconocidos (típicamente blancos, de clase media, hombres). Es necesario desde el principio entender el panorama entrelazado de los impactos de las culturas 
dominantes sobre grupos de sociedades que pueden, alguna vez, haber sido caracterizadas como del tercer mundo o dependientes, y quizás ahora puedan ser ventajosamente examinadas desde las perspectivas de la marginalidad. Ambos, el concepto médico-criminal de la homosexualidad (definida como enfermedad y/o como un crimen) y la instancia que afirma que la homosexualidad es una identidad sexual legítima (hoy, comúnmente llamada teoría queer, un término privilegiado para cubrir ambas sensibilidades, la gay y la lesbiana, en sus aspectos comunes y en sus diferencias), son términos de la sociedad dominante que tienen una aplicación imperfecta y hasta distorsionada en lo que a América Latina se refiere. Esta mala aplicación es aún más grave si tomamos en consideración las regiones, culturas, tradiciones lingüísticas, clases sociales y tradiciones étnicas y raciales vastamente diferentes de esta región. Si uno habla en forma global de América Latina, es entonces difícil evitar el ser superficial, y si uno habla acerca de sociedades específicas de América Latina, entonces existe el riesgo de la parcialización del fenómeno en el sentido en que el rango de los datos puede que no sea lo suficientemente extenso como para garantizar conclusiones persuasivas.

La divergencia que debe notarse, tanto para la homosexualidad como para una sensibilidad gay en términos de dominación extranjera, es un reconocimiento inicial de su impropiedad y también de su improbabilidad. Los términos son impropios porque describen una configuración del orden social que puede corresponder a la sociedad anglomericana, pero no a la de Latinoamérica. Aquí, el homosocialismo y la división entre vida pública y privada siempre han incluido oportunidades para las relaciones entre personas del mismo sexo, lo que no necesariamente se deriva en algo semejante a la construcción de la identidad homosexual. Uno no debe pasar completamente por alto la cuestión del substrato de culturas indígenas en América Latina y numerosos rastros de ambos: la homosexualidad ritual y el erotismo pansexual. Son rastros de sexualidad que, a la vez, complementan la heterosexualidad procreadora y resisten quinientos años de una oralidad sexual judeo-cristiana impuesta por los conquistadores españoles. Aún más, por referirnos a la historia masculina dominante, algunas de las más diversas sociedades de América Latina proveen ejemplos que han sido codificados en su producción cultural, del macho que lo hace igualmente con hombres y con mujeres sin perder un ápice de su masculinidad. La figura del maricón es reservada exclusivamente para el penetrado, pero la pregunta que ocurre con insistencia es la disyuntiva entre la ideología del macho que penetra y el maricón penetrado y cuáles pueden ser, de hecho, los detalles íntimos de sus prácticas sexuales más allá de lo exclusivamente genital.

Es plausible extraer de lo que ha sido expuesto hasta este punto algunos comentarios finales con respecto a las posibles vías de futura investigación sobre motivos lesbianos y gay en América Latina. Por un lado, existe la necesidad de reconocer una forma de hablar de la homosexualidad como enfermedad o como crimen que ha sido incorporada a la conciencia de Latinoamérica como resultado de influencias internacionales. Lo mismo se dice con respecto a cualquier concepto unificante de sensibilidad gay, aunque debería ser evidente que la fluidez esencial del último concepto puede sin ningún esfuerzo abrir los residuos escondidos del homoeroticismo prehispánico en la sociedad de América Latina que trasciende los movimientos políticos contemporáneos, para orientarse hacia el enmarcado de prácticas culturales y literarias que son supuestamente homoeróticas en formas que escapan las categorías impuestas por la sociedad dominante. Las cuestiones lesbianas y de identidad así mismo hacen eco de los parámetros de la 
sociedad internacionalizadora dominante, mientras que al mismo tiempo en forma manifiesta implican contextos que señalan una experiencia única en América Latina, comenzando por las posibles proyecciones del sistema de comadrismo. En todos estos casos, el imperativo para hacer personal la política, ya sea al nivel de textos primarios o al nivel de lecturas individuales y profesionales, provee una colaboración única al homoerotismo de América Latina que, a través de líneas ideológicas y de género, constituye su caracterización más matizada que los varios sistemas angloamericanos, en particular los del discurso médico-jurídico y de la identidad. Y es sobre las intersecciones de la una y de los otros que ahora conviene abrir el debate analítico.

\section{Bibliografía}

Acevedo, Celmar. 1985. Homosexualidad: hacia la destrucción de los mitos. Buenos Aires: Ediciones del Ser.

Almaguer, Tomás. 1991. "Chicano Men: a Cartography of Homosexual Identity and Behavior." Différences. 3(2): 75-100.

Anzaldúa, Gloria. 1987. Borderlands/La frontera. The New Mestiza. San Francisco: Spinters/Aunt Lute.

Blanco, José Joaquín. 1980. La paja en el ojo; ensayos de crítica. Puebla: ICUAP, Centro de Estudios Contemporáneos, Editorial de la Universidad Autónoma de Puebla.

Bruce-Novoa, Juan. 1986. "Homosexuality and the Chicano Novel". Confluencia. Revista hispánica de cultura y literatura. 2(1): 69-78. También 1988. En: Genvieve Fabre (ed.), 98-106.

Carrier, Joseph. 1995. De los otros; Intimacy and Homosexuality among Mexican Men. Albuquerque: University of New Mexico Press.

Cohen, Ed. 1993. Talk on the Wilde Side; Toward a Genealogy of a Discourse on Male Sexualities. New York: Routledge.

Comisión Nacional sobre la Desaparición de Personas. 1984. Nunca más: informe de la Comisión Nacional sobre la Desaparición de Personas.

Daly, Mary. 1978. Gyn/ecology: the Metaethics of Radical Feminism. Boston: Beacon Press.

Dollimore, Jonathan. 1991. Sexual Dissidence: Augustine to Wild, Freud to Foucault. Oxford: Clarendon Press. 
Doty, Alexander. 1992. Making Things Perfectly Queer: Interpreting Mass Culture. Minneapolis: University of Minneapolis Press.

Foster, David William. 1991. Gay and Lesbian Themes in Latin American Literature. Austin: University of Texas Press.

1994. "Some Proposals for the Study of Latin American Gay Culture". Cultural Diversity in Latin American Literature. Albuquerque: University of New México Press, 2571.

Foster, David William (ed.). 1994. Latin American Writers on Gay and Lesbian Themes: A Bio-critical Sourcebook. Westport: Greenwood Press.

Foster, Stephen Wayne. "Latin American Studies". Cabirian and Gay Books Bulletin. 11: 2-7, 29.

Fry, Peter. 1982. "Da hierarquia à igualdade: a construçâo histórica da homosexualidade". Para inglés ver: Identidade e política na cultura brasileira. Rio de Janeiro: Zahar.

Fry, Peter y Peter MacRae. 1991. O que é homossexualidade. Sầ Paulo: Brasiliense.

Genvieve, Fabre (ed.). 1988. European Perspectives on Hispanic Literature of the United States. Houston: Arte Público Press.

Gregorich, Luis. 1985. Literatura y homosexualidad y otros ensayos. Buenos Aires: Editorial Legasa.

Hazen, Dan C (ed.). 1985. Latin American Masses and Minorities. Madison: SALALM Secretariat, Memorial Library, University of Wisconsin.

Howes, Robert. 1985. "The Literature of Outsiders: the Literature Of the Gay Community en Latin America". En: Dan C. Hazen (ed.), 288-304, 580-91.

Jáuregui, Carlos Luis. 1978. La homosexualidad en la Argentina. Buenos Aires: Ediciones Tarso.

Jockl, Alejandro. 1984. Ahora, los gay. Buenos Aires: Ediciones de la Pluma.

Katz, Jonathan. 1995. The Construction of Heterosexuality. New York: Dutton.

Leyland, Winston (ed.). 1979. Now a Volcano: an Antology of Latin American Gay Literature. Traducido por Erskine Lane, Franklin D. Blanton, Simon Karlinsky. San Francisco: Gay Sunshine Press. 
1983. My Deep Dark Pain is Love, a Collection of Latin American Gay Fiction. San Francisco: Gay Sunshine Press.

Lima, Délcio Monteiro de. 1983. Os homoeróricos. Rio de Janeiro: Francisco Alves.

Lumsden, Ian. 1991. Homosexualidad: sociedad y estado en México. México D.F.: Solediciones; Toronto: Canadian Gay Archives.

1996. Machos, Maricones and Gays; Cuba and Homosexuality. Philadelphia: Temple University Press.

Martin, Robert K. 1993. “Roland Barthes: toward an Écriture gaie”. En: David Bergman (ed.), 282-98.

Mattoso, Glauco. 1986. Manual do pedólatra amador, aventuras \& leituras de um tarado por pés. São Paulo: Editora Expressão.

Monsiváis, Carlos. 1971. Días de guardar. México D. F.: Era.

1977. Amor Perdido. México D. F.: Era.

1988. Escenas de pudor y livianidad. México D. F.: Grijalbo.

Mott, Luiz. 1987. O lesbianismo no Brasil. Porto Alegre: Mercado Alberto.

1988. Escravidăo, homossexualidade e demonologia. Săo Paulo: Icone.

1988. O sexo proibido: virgens, gays e escravos nas garras da Inquisiçăo. Campinas: Papirus Editoras.

Murray, Stephen O. (ed.). 1987: Male Homosexuality in Central and South America. San Francisco: Instituto Obregón; Nueva York: GAU-NY.

Núñez Noriega, Guillermo. 1994. Sexo entre varones: poder y resistencia en el campo sexual. Hermosillo: El Colegio de Sonora, División de Ciencias Sociales.

Paker, Richard G. 1990. Bodies, Pleasures, and Passions: Sexual Culture in Contemporary Brazil. Boston: Beacon Press.

Puig, Manuel. 1990. “El error gay”. El porteño. Setiembre: 32-3.

Ramos, Juanita (ed.). 1987. Compañeras: Latina Lesbians (an Antology). New York: Latina Lesbian History Project. 
Rich, Adrienne. 1986. "Compulsory Heterosexuality and Lesbian Existence". En: Blood, Bread, and Poetry: Selected Prose 1979-1985. Nueva York: W. W. Norton, 23-75.

Ruiz Esparza, Jorge. 1990. "Homosexualidad: la diferencia y la escritura". En: Coloquio internacional: escritura y sexualidad en la literatura hispano-americana. Poitiers: Université de Poitiers, Centre de Recherches Latino-Americaines.

Russo, Vito. 1987. The Celluloid Closet: Homosexuality in the Movies. Nueva York: Harper and Row.

Schaefer-Rodríguez, Claudia. 1989. "The Power of Subversive Imagination: Homo-sexual Utopian Discourse in Contemporary Mexican Literature". Latin American Literary Review. 33: 29-41.

Schneider, Luis Mario. 1985. "El tema homosexual en la nueva narrativa mexicana". Casa del tiempo [México]. 49-50: 82-6.

Schwartz, Kessel. 1975. "Homosexuality as a Theme in Representative Contemporary Spanish Americans Novels". Kentucky Romance Quarterly. 22: 247-57.

Sedgwick, Eve Kosofsky. 1990. Epistemology of the Closet. Berkeley: University of California Press.

Silverman, Kaja. 1992. Male Subjectivity at the Margins. Nueva York: Routledge.

Soto Francisco. 1991. "Reinaldo Arena's Literary Legacy”. Christopher Street. 156:12-6.

Trevisan, Joâo S. 1986. Perverts in Paradise. Trad. Martin Foreman. London: GMP Publications.

Trujillo, Carla (ed.). 1991. Chicana Lesbians: the Girls Our Mothers Warned Us about. San Francisco: Aunt Lute Books.

Villanueva, Alfredo. 1976. "Machismo vs. Gayness: Latin American Fiction”. Gay Sunshine. 29-30: 22.

Young, Allen. 1981. Gays under the Cuban Revolution. San Francisco: Grey Fox Press. 